\title{
Off-line Signature Verification Using an Enhanced Modified Direction Feature with Single and Multi-classifier Approaches
}

\section{Introduction}

iv ithin the field of human identification, the usage of biometrics is growing because of its unique properties such as hand geometry, iris scanning and fingerprint or DNA analysis. The use of signatures has been one of the most convenient methods for the identification and verification of human beings. One of the challenges of

\section{One of the challenges of signature verification is that a signature may change depending on behavioral characteristics such as mood, fatigue, etc.}

approaches, such as that based on fuzzy modeling and the employment of the Takagi-Sugeno model, have been proposed using angle features extracted from a box approach to verify and identify signatures [4]. Also, the GSC (Gradient, Structural and Concavity) feature extractor provided results as high as $78 \%$ for verification and 93\% for identification [7]. Various classifiers, such as Support Vector Machines (SVMs) and Hidden Markov Models (HMMs), have also been successful in off-line signature verification, with SVMs providing an overall better result than the HMM-based approach [6]. Research into person identification and verifica-

signature verification is that a signature may change depending on behavioral characteristics such as mood, fatigue, etc. The signature is termed a behavioral biometric when affected by behavior.

The challenging aspects of automated signature verification have been a true motivation for researchers. Research into signature verification has been vigorously pursued for a number of years [3] and is still being explored, particularly in the off-line mode [1], [11]. On-line signature verification is differentiated from off-line verification by the number of features readily available for analysis. The number of features which may be extracted from on-line mediums typically exceed those obtained through off-line verification. For example, time, pressure, and speed can be extracted from on-line modes of verification [8]. Previous tion, including physical traits, fingerprints, and signature analysis has also been investigated [12].

In the field of pattern recognition, choosing a powerful set of features is crucial for both the application and the classifier. Lv et al. used the direction distribution, stroke width distribution, grey distribution, and moment feature to conduct signature verification [10].

Previous work using the Modified Direction Feature (MDF) generated encouraging results, reaching an accuracy of up to $90.24 \%$ for cursive

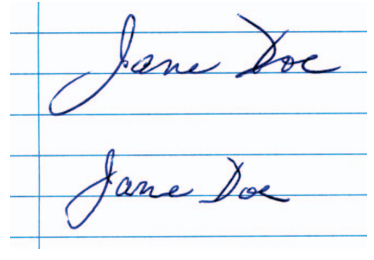
handwritten character recognition [9]. As an extension to previous work, the research in this paper adapts, extends, and investigates MDF with signature images. Specifically, a number of features have been combined with MDF to capture various structural and geometric properties of the signatures being investi- gated. The verification process implies the usage of forged signatures, discriminating the genuine from the forged. In this paper, we present experimental results for signature verification using MDF and propose some modifications of the features extracted.

\section{Methodology}

To perform verification or identification of a signature, several steps must be undertaken. After preprocessing all signatures from the database by converting them to a portable bitmap (PBM) format, their boundaries are defined to facilitate the extraction of features using MDF. Verification experiments are then performed with neural-based classifiers. Figure 1 illustrates the signature verification process.

\subsection{Signature Database}

Experiments have been performed by combining the "Grupo de Procesado Digital de Senales" (GPDS) signature database [2] and additional signatures collected by the authors. The results provided in this research used a total of 2376 signatures.

From those 2376 signatures, we used 44 sets of signatures; and, for each set, 24 samples of genuine and 30 samples of forgeries were available. Samples of the genuine and forged signatures are displayed in Figure 2.

\subsection{Boundary Extraction}

The boundary of each signature must be extracted prior to the feature extraction process. The binary image of each signature is processed, and the contour is extracted, providing the first step in the process of reducing the amount of data describing each pattern. Figure 3 shows the extraction of a signature's boundary [9]. 


\subsection{Feature Extraction}

The features extracted must be appropriate for both the application and the classifier used. MDF and additional features are proposed to provide pertinent information for the signature verification problem. Shape features and geometrical features are commonly extracted from signature images. Huang et al. described a method distinguishing these two kinds of features [5].

\subsubsection{The Modified Direction Feature}

Related work using MDF is described in Liu and Blumenstein [9]. This technique employs a hybrid of two other feature extraction techniques, Direction Feature (DF) and the Transition Feature (TF).

DF extracts Direction Transitions (DT), based on the replacement of the foreground pixels by their direction values, which are categorized by five different direction numbers: vertical direction value $=2$, right diagonal value $=3$, horizontal direction value $=4$, left diagonal value $=5$ and intersections $=9$ The feature vector is extracted by zoning and computing the most representative direction values in a given zone.

TF records the Locations of the Transitions (LTs) between foreground (1s) and background (0s) in binary digital images. The image is traversed from the following directions: left to right, right to left, top to bottom, and bottom to top. Each time a change from ' 0 ' to ' 1 ' or from ' 1 ' to ' 0 ' occurs, the ratio between the location of the transition and the length/width of the image traversed is recorded as a feature. An averaging algorithm is used to obtain a feature vector of appropriate size in order to decrease the training/classification time. These are based on the extraction technique present in TF.

Not only are the locations of the transitions calculated, but also the corresponding direction values are determined, facilitating the storage of LTs and DTs. The width to height ratio feature was also included to comprise MDF.

\subsubsection{The Centroid Feature}

Another feature was considered in this research relating to the dominant angle

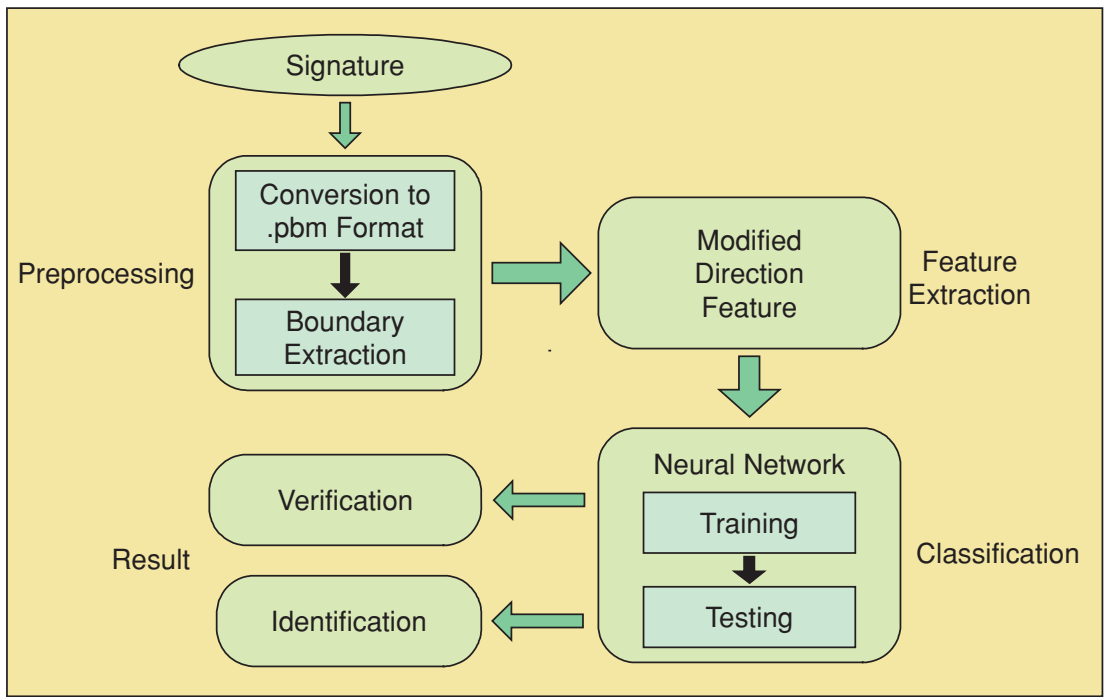

FIGURE 1 Procedure to identify/verify a signature from a database.

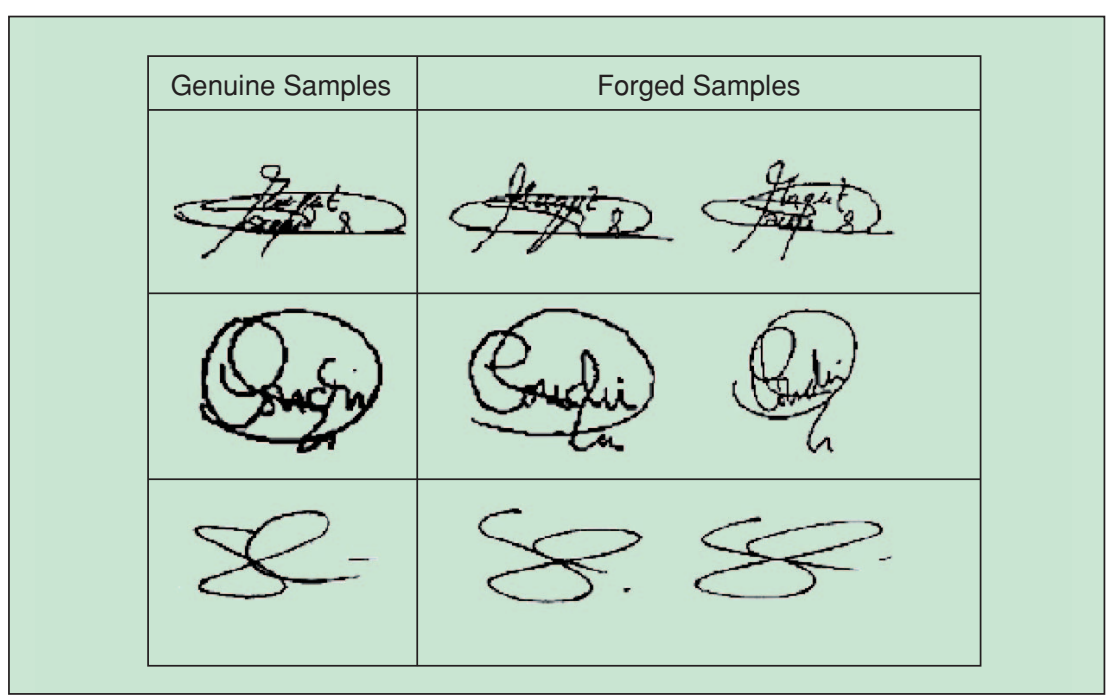

FIGURE 2 Signature samples from the database.

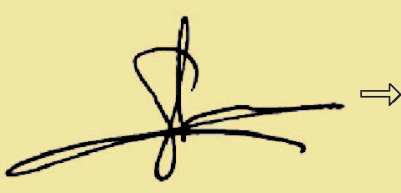

(a)

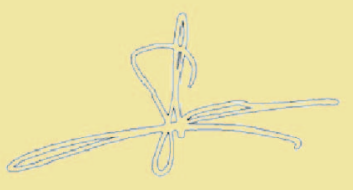

(b)

FIGURE 3 Boundary extraction example from a signature: (a) original signature, (b) following boundary extraction.

of the signature's pixel distribution, given by its 'centroids'.

First, the signature image was separated into two equal parts. The position of the centre of gravity in each part was calculated (A and B in Figure 4). The angle between the horizontal axis and the line obtained by linking the two centers of gravity was the feature added. To convert this angle into 


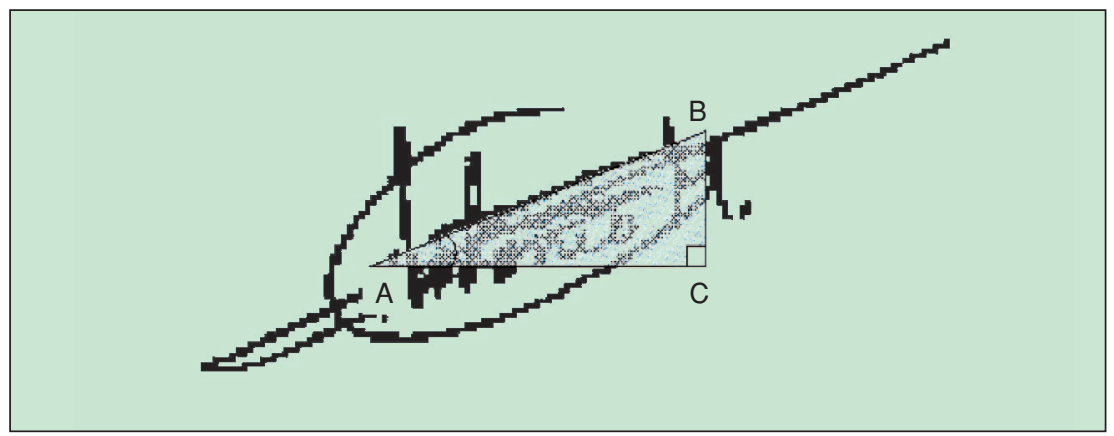

FIGURE 4 Centroid feature.

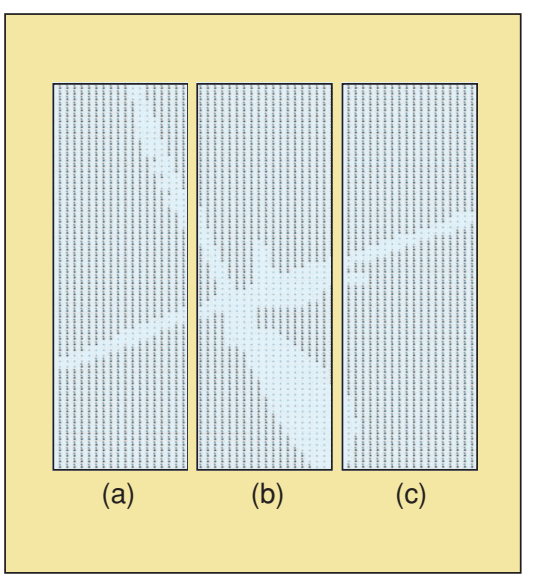

FIGURE 5 TriSurface feature.

the range of 0 to 1 (making it appropriate for use with a neural classifier), calculations were performed with the coordinates of the centers of gravity. A right triangle was considered using the centers of gravity as the extreme points of its hypotenuse, the row of the first centre of gravity and the column of the other, intersecting to form the right angle.

To obtain the 'centroid' feature, the angle $\alpha$ was first considered in the left part of the triangle, and then a calculation was performed to normalize the value to a range of 0 to 1 . Equations (1) and (2) are applied to get this value. The concept is depicted in Figure 4 . The angle $\alpha$ is illustrated at point A.

$$
\begin{aligned}
\alpha & =\frac{\arcsin \left(\frac{\text { height }}{\text { hypotenuse }}\right)}{\pi} \\
\text { centroid } & =\alpha+\frac{1}{2}
\end{aligned}
$$

\subsubsection{The TriSurface Feature}

The surface area of two visually different signatures could be the same. For the purpose of increasing the accuracy of a feature describing the surface area of a signature, the 'triSurface' feature was investigated as an extension in which the signature was vertically separated into three equal parts. The surface area feature is the surface covered by the signature, including the holes contained in it. The total number of pixels in the surface was tallied, and the proportion of the signature's surface over the total surface of the image was calculated. This process was used for the three equal parts of the signature, giving three values between 0 and 1 . Figure 5 illustrates this concept.

\subsubsection{The Length Feature}

The length feature represents the length of the signature after scaling all the signatures from the database to the same height. To normalize the value between 0 and 1 , the minimum signature length obtained in the whole database was considered as 0 , and the maximum signature length was considered to be 1 . The remaining signature lengths were then converted to values between this minimum and maximum range.

\subsubsection{The Sixfold-Surface Feature}

This feature is different from the TriSurface feature mainly in two ways. Firstly, the number of feature values obtained is doubled to six with the Sixfold Surface. Secondly, centers of gravity are determined to assist in the calculation of the Sixfold Surface features.

The signature image is first divided into three parts vertically. The center of gravity is calculated for each of the three parts, and the signature surface above and below the horizontal line of the center of gravity (giving two subsections for each part) was calculated. The result was a set of six feature values corresponding to the surface of the six sub-sections as illustrated in Figure 6.

\subsubsection{The Best-Fit Features}

The line of best fit usually attempts to represent a scatter of points in an area. In order to obtain an approximation for the signature's skew, the line of best fit was calculated using minima and maxima from the bottom edge of the signature. Similarly the line of best fit from the top of the signature was also calculated. The angles between each of these lines and the $\mathrm{X}$-axis were calculated, giving two features. The surface area enclosed between the two lines became the third feature. Figure 7 depicts the concept.

To calculate the correlation coefficient and the line of best fit, we used the following formulae:

$$
\begin{aligned}
r_{X Y} & =\frac{\sum x y / N-\left(M_{X} \times M_{Y}\right)}{S_{X} \times S_{Y}} \\
b_{X Y} & =r_{X Y} \frac{S_{Y}}{S_{X}} \\
a & =M_{Y}-b_{X Y} M_{X} \\
y & =a=b_{X Y} x
\end{aligned}
$$

Equation (3) is the raw formula used to obtain the correlation coefficient. $M_{X}$ and $M_{Y}$ are the means of the set of pixels obtained from the minima and maxima. $S_{X}$ and $S_{Y}$ are the respective standard deviations. $\mathrm{x}$ and $\mathrm{y}$ are the coordinates of the pixels, and $\mathrm{N}$ is the total number of pixels selected.

To obtain the line of best fit, we needed to calculate the slope of the regression, and also to determine where to place the line on the signature. The slope was calculated using Equation (4) and then by multiplying the correlation between the two variables by the standard deviations of $\mathrm{X}$ and $\mathrm{Y}$.

Equation (5) was the formula used to determine the intersection at the $\mathrm{Y}$-axis. That is, it is the point where the regression line crosses the $\mathrm{Y}$-axis at $\mathrm{X}=0$. 


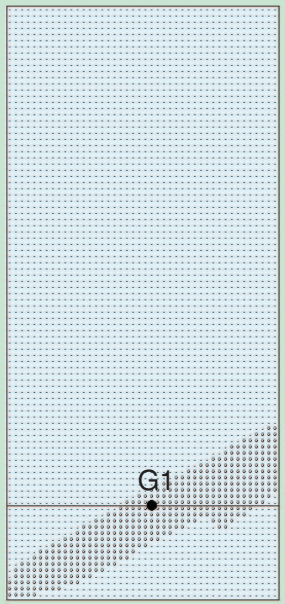

(a)

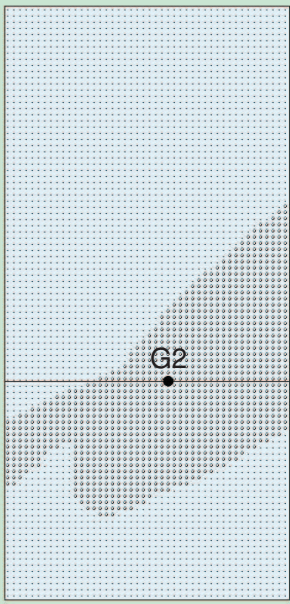

(b)

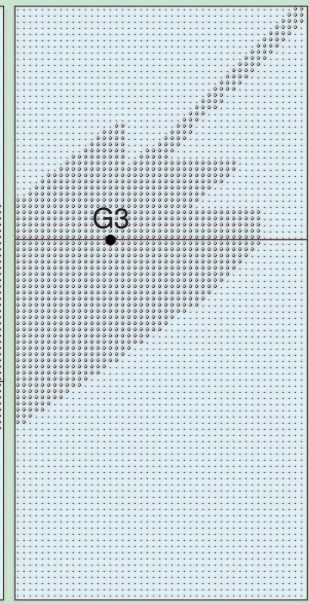

(c)

FIGURE 6 Six Fold Surface feature. G1, G2 and G3 are the centers of gravity for the respective sections (a), (b) and (c).

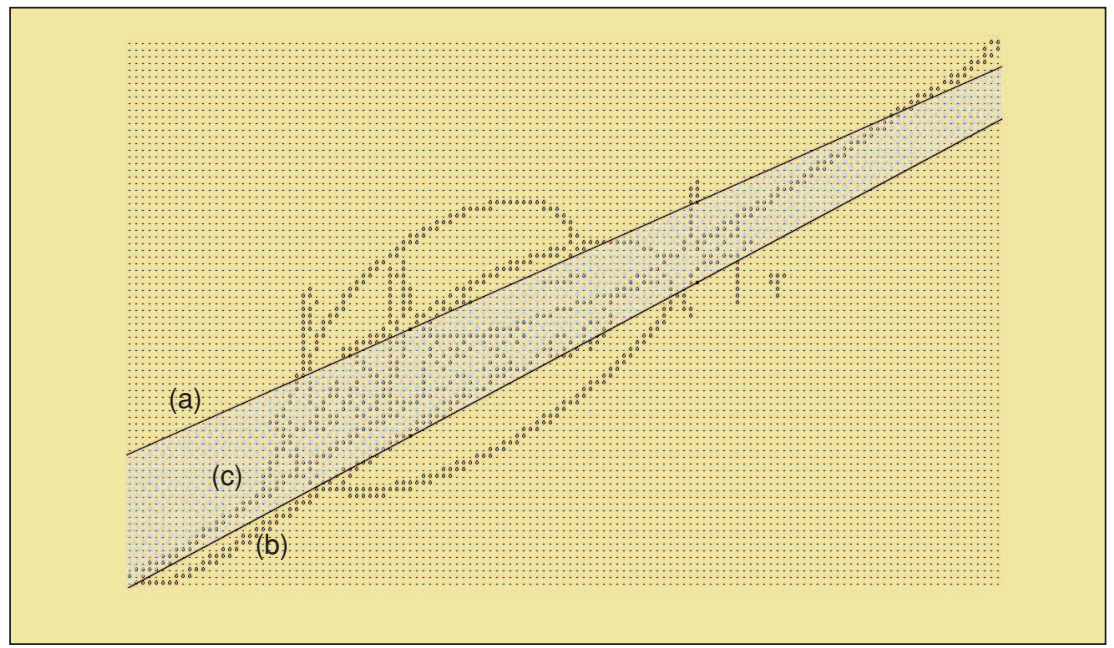

FIGURE 7 Best-Fit features. (a) and (b) lines of best fit (top and bottom), (c) surface between the two lines.

Equation (6) is the formula for the line of best fit, based on the previous equations (3), (4) and (5).

\subsection{Neural Network Architectures}

Two neural network classifiers were used to verify the signatures: the Resilient Backpropagation (RBP) neural network and the Radial Basis Function (RBF) network.

Depending on the purpose of the verification, it could be justified to use a single network for the whole database, or one neural network for each signature. The usage of a single neural network in our method not only verified the signa- tures (i.e. it accepted or rejected the signature), but it also identified the owners of the signatures presented to the network. This architecture would be used most often to verify a signature from a cheque or a signed document. Multiple classifiers (i.e. neural networks) might be used in a situation where a second confirmation would be needed from an individual after the usage of a primary form of identification. Experiments using both configurations were conducted.

\subsubsection{Single Neural Network}

The database was split into two parts to perform the training and testing compo- nents, using a single neural network. From the genuine set, 20 samples of each signature were used for training, and 4 for testing. We used 25 samples of each signature for training the forged signatures and 5 for testing purposes. Parameters such as the number of iterations and hidden units were varied extensively for RBP during experimentation; also, the number of centers was varied between 40 and 10000 for the RBF network. Both of the classifiers used 45 outputs to classify the signatures: 44 outputs for each different set of signatures, and the last output for the forgeries.

\subsubsection{Multiple Neural Networks}

In their paper, Ferrer et al. used a system in which a signature was verified using a decision threshold $\tau$ ( $i$ ) [2].

When a signature was to be verified, the classifier appropriate to the claimed signature was used. Each classifier used 2 outputs, accepting or rejecting a signature. The use of multiple neural networks for verification in this research is similar to the concept described in the work by Ferrer et al.

Table 1 presents the composition of the training and testing sets for this configuration. Updating a system using multiple neural networks is convenient since the addition of a new signature would not necessarily require the re-training of the previous neural networks.

\section{Experiments}

The signatures were first converted into a Portable Bitmap (PBM) format in order to facilitate processing as binary images. Subsequently, their boundaries were extracted for further processing. An adapted version of MDF (including the new features described in the previous sub-sections) was used for feature extraction. The features were values scaled between 0 and 1 to facilitate neural network training for experimentation. Java, C, and Matlab were used for implementing and investigating the proposed techniques.

\subsection{Verification Experiments}

Using MDF-CTLFS (a combination of MDF with all features described in the 


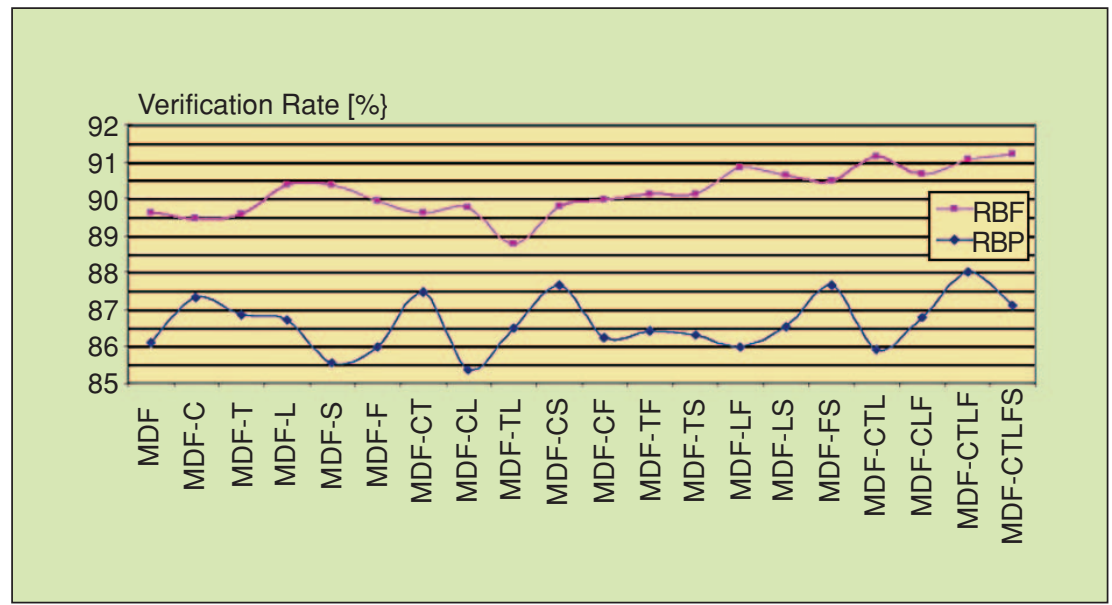

FIGURE 8 Comparison of RBP and RBF.

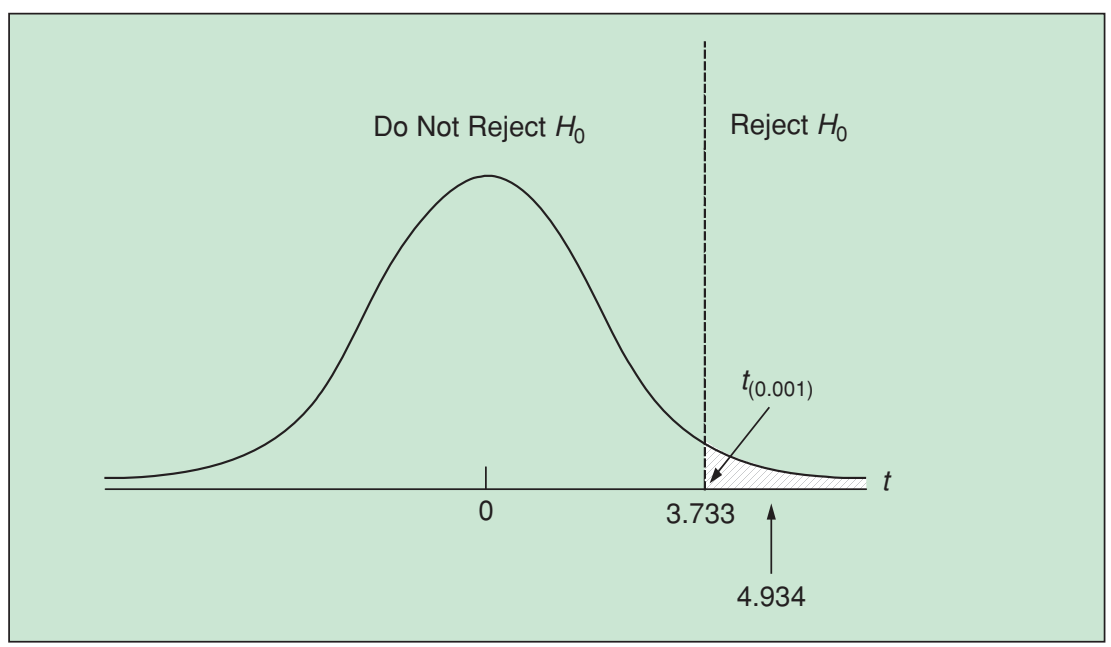

FIGURE 9 Area of the right-tailed hypothesis: $t>t_{0.001}$.

previous section), a total of 135 feature values were obtained. Modifications of the MDF technique have been proposed to improve classification accuracy, with the hypothesis that adding distinguishing features could improve the verification rate. Experiments with MDF merged with each new feature or a combination of new features were conducted separately, increasing the feature vector up to 135 inputs.

The usage of a single neural network implies that re-training should be performed if any new signatures are to be added in the database. However, in our method, identification is also performed whilst verifying the signature. In other words, the classifier is not only accepting or rejecting a signature, but is also identifying the owner. Conversely, using a single neural network for each signature (multiple networks) assumed that the signature's owner is already known. We could assume that an ID card, for example, or any other identification means might be used primarily; and then the owner would have to sign to confirm his/her identification, instead of entering a key/password.

\subsection{Six-fold Validation}

Six-fold cross validation was used in this research. The signatures from the database were partitioned into six equal sections in their related type (genuine or forged). Thus, six sections for the genuine, and six sections for the forged, resulted from this partition. Five sec-
TABLE 1 Set composition for training and testing each signature.

TRAINING TESTING

\begin{tabular}{lrr} 
GENUINE SIGNATURE & 20 & 4 \\
RANDOM GENUINE & 172 & 860 \\
FORGERIES & 5 & 25 \\
\hline TOTAL & 197 & 889
\end{tabular}

tions of each were used for training, and testing was performed with the remaining unseen signature sets. As a result, six training and testing sets were created; and experiments were run six times with the different sets, obtaining varying results. By doing so, the results for signature verification were suitably validated.

\section{Results and Discussion}

This section describes the results obtained by either employing MDF in its original version, or with MDF accompanied by one or more of the following extra features: centroid (C), triSurface (T), length (L), sixfold-surface (S) and the best-fit (F).

In the first sub-section (of those that follow), with the single neural network, verification was achieved by performing identification simultaneously. To satisfy the six-fold validation process, the experiments were conducted using 6 different configurations on the signatures for the training and testing sets.

In the second sub-section, using the multiple neural networks, several other experiments needed to be conducted. Six-fold validation was also applied, and a classifier was trained for each signature.

The verification process is different from identification, as forged signatures are also part of the database in the former; the classifier was required to distinguish between genuine and forged signatures. Optimally, results should show a high acceptance rate for the genuine and a high rejection rate for forged signatures. In the following experiments, 1560 signatures for training and 546 signatures for testing were used to conduct verification.

As shown in Table 4 and Table 5, MDF merged with other features outperformed the original version of MDF 
for most of the experiments. Using $\mathrm{RBP}$, the best result obtained reached an $88 \%$ verification rate (v. r.), with MDF-CTLF. Using RBF, MDFCTLFS obtained the best result, reaching $91.21 \%$ v. r. Figure 8 illustrates the comparison between RBF and RBF.

\subsection{Single Neural Network}

Using the RBF classifier, the results obtained were better overall in comparison with the RBP classifier. Using different algorithms, and having different configurations, the comparison between $\mathrm{RBP}$ and RBF is made only on the basis of the best results obtained from the experiments conducted. Table 2 displays the results obtained. The importance of the cross validation is shown since the results varied significantly depending on the sets.

On average, RBF performed better than $\mathrm{RBP}$, reaching a verification accuracy of $89.77 \%$. It might however be noted that the top verification rate was obtained with RBP (93.69\%) whereas the top verification rate using RBF was $92.17 \%$.

Our system compared well with another system described recently in the literature, which provided an error rate of $18.50 \%$ on a smaller signature database, using the radial basis function with support vector machines [10].

\subsection{Multiple Neural Networks}

The results obtained, using multiple neural networks were significantly higher compared to the single network. This method is also faster to train and test.

Where a single neural network could take minutes to perform the training and testing phases, the usage of multiple neural networks significantly decrease this phase, with just a few seconds to finish each training and testing operation.

Contrary to the results obtained using the single classifier configuration, RBP outperformed RBF using multiple classifiers, achieving an overall verification error rate of $1.16 \%$ (Table 3 ), upon averaging the error rates of the different signatures. This result is comparable to the error rate of $2.2 \%$ reported in [2], using Hidden Markov models, although on a bigger database.

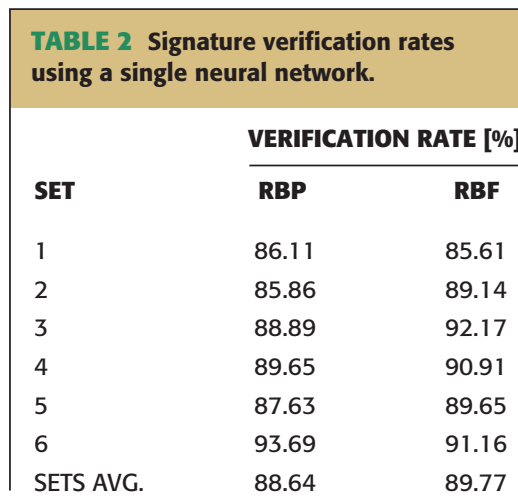

\subsection{Comparison between MDF and MDF-CTLFS using RBF (Single Network)}

In order to evaluate which of the feature configurations were the most successful between MDF and MDF-CTLFS (MDF combined with all the features investigated), it is possible to directly compare the best results obtained using a single network. As shown in Table 4, MDF provided a verification rate of $89.61 \%$, and MDF-CTLFS gave a verification rate of $91.21 \%$. However, it is not accurate to conclude that MDFCTLFS is definitely better than MDF, based only on these figures. Another way is to conduct hypothesis testing.

By observing the results obtained from identical network configurations, MDF-CTLFS performed better in terms of providing a better recognition rate. It is hypothesized that the population mean recognition rate of MDFCTLFS is higher than the population mean recognition rate of MDF.

Hypothesis testing is formulated as such: let $\mu_{d}$ be the population mean verification accuracy between MDF and MDF-CTLFS; let $\bar{d}$ be the sample mean of the difference between MDF and MDF-CTLFS. The testing was performed at a $0.1 \%$ significance level using the matched pairs method. Let X1 denote each data point obtained by MDF-CTLFS and X2 each data point from MDF.

Important figures can be obtained from the matched pairs. They are 1) the number of data points, denoted by $n, 2$ ) the difference between two matched pairs, $\mathrm{d}=\mathrm{X} 1-\mathrm{X} 2,3)$ the sample standard deviation of the differences

\begin{tabular}{|c|c|c|}
\hline \multirow[b]{2}{*}{ SET } & \multicolumn{2}{|c|}{$\begin{array}{l}\text { VERIFICATION } \\
\text { ERROR RATE [\%] }\end{array}$} \\
\hline & RBP & RBF \\
\hline 1 & 1.21 & 1.20 \\
\hline 2 & 1.15 & 1.03 \\
\hline 3 & 1.16 & 1.31 \\
\hline 4 & 1.19 & 1.10 \\
\hline 5 & 1.09 & 1.35 \\
\hline 6 & 1.16 & 1.31 \\
\hline SETS AVG. & 1.16 & 1.22 \\
\hline
\end{tabular}

between two sets of data. The difference between each matched pair value is displayed in Table 6 .

16 data points were taken to perform the calculation, and a result of 1.454 was found for the sample mean.

$$
\begin{aligned}
\bar{d} & =1.454 \\
S_{d} & =\sqrt{\frac{\sum(d-1.454)^{2}}{n-1}} \\
S_{d} & =1.178
\end{aligned}
$$

And the hypothesis testing steps are:

Step 1) $\mathrm{H}_{0}: \mu_{d}=0$

$$
\mathrm{H}_{a l t}: \mu_{d}>0
$$

Step 2) $\mathrm{n}+16, \bar{d}=1.454, S_{d}=1.178$ Step 3) Critical values:

Degrees of freedom $=15$

$t_{0.001}=3.733$

Step 4) Test statistic

$$
\begin{aligned}
t & =\frac{\bar{d}-\mu_{d}}{S_{d} / \sqrt{n}}=\frac{1.454-0}{(1.178 / \sqrt{1} 6)} \\
t & =4.943
\end{aligned}
$$

Step 5) According to the calculation, $\mathrm{t}$ was found superior to t0.001, as shown in Figure 9. The statistical test used, provides sufficient evidence to conclude that MDF-CTLFS has a higher verification accuracy on average than MDF (for the database used) at a $0.1 \%$ significance level. In other words, there is $99.9 \%$ confidence that on average, MDFCTLFS outperforms MDF.

\subsection{Analysis of the Rates Obtained with MDF-CTLFS (Single Network)}

An advantage of using 45 outputs (44 for the genuine set of signatures, one for 
the forged sets) for neural verification is the possibility of analyzing the output obtained following experimentation. Some observations may be made as a result of experimentation with the highest performing feature and classifier combination (MDF-CTLFS and RBF).

Amongst the genuine signatures, on average, $88.68 \%$ of these were correctly verified, and $93.59 \%$ forged were correctly rejected. No more than $12 \%$ of genuine signatures were rejected as forged signatures. A maximum of $3 \%$ of the genuine signatures were misclassified as another genuine category. From one perspective, it could be preferable to have a signature rejected rather than misclassified as another genuine signature.

Amongst the forged signatures, an average of $93.59 \%$ were correctly rejected. $4.49 \%$ was the maximum rate for the forgeries misclassified as their genuine signature equivalents. A maximum of $3.85 \%$ were misclassified as another genuine signature.

\subsection{Comparison of Verification Rate with Other Researchers}

It is difficult to compare the results obtained in this paper with those of other researchers, as many have used different databases for experimentation. However, a general comparison can be performed with the researchers that proposed the GPDS database. As mentioned previously, the database used in the current research contains 39 sets of signatures from the GPDS database; this was the only subset available when the experiments were conducted. The full GPDS database contains a total of 160 sets of signatures.

The proposed system, using a single neural network classifier for training and testing, reached a verification accuracy of $91.21 \%$. In their research, Martinez et al. obtained their best results with HMMs, showing a $2.2 \%$ error rate using random forgeries, and a $14.1 \%$ error rate using simple forgeries. The objective of their verification system was to differentiate the genuine from the forgeries. Using a random forgery meant that the signature sample belonged to a writer,

\begin{tabular}{|c|c|c|c|c|c|}
\hline & \multicolumn{5}{|c|}{ VERIFICATION RATE [\%] } \\
\hline & SET 1 & SET 2 & SET 3 & SET 4 & SETS AVG. \\
\hline MDF & 83.33 & 88.64 & 87.18 & 85.16 & 86.08 \\
\hline MDF-C & 84.07 & 87.91 & 89.56 & 87.73 & 87.32 \\
\hline MDF-T & 85.53 & 86.45 & 87.55 & 87.91 & 86.86 \\
\hline MDF-L & 84.43 & 88.10 & 89.01 & 85.35 & 86.72 \\
\hline MDF-S & 83.88 & 83.52 & 88.46 & 86.26 & 85.53 \\
\hline MDF-F & 83.33 & 87.36 & 88.28 & 84.98 & 85.99 \\
\hline MDF-CT & 84.43 & 87.36 & 88.46 & 89.56 & 87.45 \\
\hline MDF-CL & 82.23 & 87.73 & 84.98 & 86.45 & 85.35 \\
\hline MDF-TL & 82.97 & 86.63 & 87.18 & 89.19 & 86.49 \\
\hline MDF-CS & 84.62 & 89.01 & 88.28 & 88.64 & 87.64 \\
\hline MDF-CF & 83.15 & 88.64 & 87.18 & 85.90 & 86.22 \\
\hline MDF-TF & 83.52 & 87.00 & 87.91 & 87.18 & 86.40 \\
\hline MDF-TS & 79.85 & 89.01 & 88.46 & 87.91 & 86.31 \\
\hline MDF-LF & 83.15 & 86.63 & 87.00 & 87.18 & 85.99 \\
\hline MDF-LS & 83.70 & 88.46 & 88.83 & 85.16 & 86.54 \\
\hline MDF-FS & 87.36 & 87.91 & 88.64 & 86.63 & 87.64 \\
\hline MDF-CTL & 81.68 & 87.36 & 87.36 & 87.18 & 85.90 \\
\hline MDF-CLF & 83.15 & 87.36 & 89.19 & 87.36 & 86.77 \\
\hline MDF-CTLF & 87.36 & 87.73 & 89.74 & 87.18 & 88.00 \\
\hline MDF-CTLFS & 83.52 & 86.63 & 90.66 & 87.55 & 87.09 \\
\hline
\end{tabular}

\begin{tabular}{|c|c|c|c|c|c|}
\hline & \multicolumn{5}{|c|}{ VERIFICATION RATE [\%] } \\
\hline & SET 1 & SET 2 & SET 3 & SET 4 & SETS AVG. \\
\hline MDF & 87.91 & 90.48 & 89.74 & 90.29 & 89.61 \\
\hline MDF-C & 88.10 & 89.74 & 90.11 & 89.93 & 89.47 \\
\hline MDF-T & 87.73 & 90.48 & 89.93 & 90.11 & 89.56 \\
\hline MDF-L & 89.01 & 91.21 & 90.66 & 90.66 & 90.38 \\
\hline MDF-S & 89.38 & 90.11 & 91.03 & 91.03 & 90.38 \\
\hline MDF-F & 88.28 & 90.66 & 90.11 & 90.66 & 89.93 \\
\hline MDF-CT & 88.28 & 90.29 & 90.11 & 89.74 & 89.61 \\
\hline MDF-CL & 88.10 & 90.84 & 89.74 & 90.29 & 89.74 \\
\hline MDF-TL & 89.38 & 87.91 & 89.38 & 88.46 & 88.78 \\
\hline MDF-CS & 88.10 & 90.29 & 90.66 & 90.11 & 89.79 \\
\hline MDF-CF & 88.28 & 91.39 & 90.11 & 90.11 & 89.97 \\
\hline MDF-TF & 88.10 & 90.29 & 90.84 & 91.21 & 90.11 \\
\hline MDF-TS & 88.46 & 90.84 & 90.29 & 90.84 & 90.11 \\
\hline MDF-LF & 89.38 & 91.21 & 90.84 & 91.94 & 90.84 \\
\hline MDF-LS & 89.74 & 91.03 & 90.48 & 91.21 & 90.61 \\
\hline MDF-FS & 90.11 & 89.93 & 90.84 & 91.03 & 90.48 \\
\hline MDF-CTL & 89.19 & 91.76 & 91.94 & 91.58 & 91.12 \\
\hline MDF-CLF & 88.83 & 91.58 & 90.84 & 91.39 & 90.66 \\
\hline MDF-CTLF & 88.10 & 91.94 & 91.94 & 92.31 & 91.07 \\
\hline MDF-CTLFS & 88.83 & 92.31 & 91.94 & 91.76 & 91.21 \\
\hline
\end{tabular}

which was different from those used in the signature model. In this paper, each single signature from the testing set was presented to the trained neural classifier for verification. Consequently, a tested signature, genuine or forged, is not assumed to be known before being classified. Hence, although it is difficult to directly compare the results in this paper with those presented by Martinez et al. (due to the difference in experimental methodology and database size), it is possible to observe that the results are favorable in comparison. Ferrer et al. obtained an error rate of 2.2\% using Hidden Markov Models, on a bigger database. However, it is interesting to compare this result with the verification error rates of $1.16 \%$ with RBF and $1.22 \%$ with RBP that we obtained from our experiments. 


\begin{tabular}{|lcr}
\hline \multicolumn{3}{|l}{ TABLE 6 Obtaining values for } \\
hypothesis testing. \\
X1 & $\mathbf{X 2}$ & $\mathbf{d}=\mathbf{X 1}-\mathbf{X 2}$ \\
\hline 84.07 & 82.42 & 1.65 \\
86.45 & 86.08 & 0.37 \\
87.36 & 87.55 & -0.18 \\
87.00 & 87.00 & 0.00 \\
87.73 & 86.81 & 0.92 \\
90.29 & 89.01 & 1.28 \\
91.94 & 88.46 & 3.48 \\
89.74 & 86.81 & 2.93 \\
88.10 & 87.91 & 0.18 \\
91.58 & 91.21 & 0.37 \\
92.86 & 90.11 & 2.75 \\
92.67 & 89.56 & 3.11 \\
88.83 & 87.91 & 0.92 \\
92.31 & 90.48 & 1.83 \\
91.94 & 89.74 & 2.20 \\
91.76 & 90.29 & 1.47 \\
& & \\
\hline
\end{tabular}

\section{Conclusions}

The principal objective of this paper was to investigate the efficiency of the enhanced version of the MDF feature extractor for signature verification. Investigations adding new feature values to MDF were performed, assessing the impact on the verification rate of the signatures, using six-fold cross validation. Two different neural classifiers were used and two methodologies for verification were applied. The experiments conducted, whereby MDF was merged with the new features, provided very encouraging results.

Using RBP, MDF reached an $86.08 \%$ v. r., and MDF-CTLF reached $88 \%$ v. r. The RBF classifier provided better results than the RBP classifier overall with a single network configuration. The best v. r. obtained reached 91.21\% with MDF-CTLFS, the combination of all the features described in this paper. However, with the multi-network configuration, RBP outperformed $\mathrm{RBF}$ with an error rate of $1.16 \%$.

In future research, investigations will be conducted to enhance the feature extraction process. These include further combinations and investigations of the features. In addition, a larger signature database will be collected, including multilingual signatures, to investigate the techniques proposed in this paper. Additional classifiers, including Support Vector Machines (SVMs), will also be investigated for verifying the signatures.

\section{References}

[1] S. Chen and S. Srihari, "Use of exterior contour and shape features in off-line signature verification," 8th International Conference on Document Analysis and Recognition
(ICDAR '05), pp. 1280-1284, 2005

[2] M.A. Ferrer, J.B. Alonso, C.M. Travieso, "Offline geometric parameters for automatic Signature Verification using fixed-point arithmetic," IEEE Transactions on Pattern Analysis and Machine Intelligence, vol. 27, no. 6, pp. 993997, 2005.

[3] K. Han and I.K. Sethi, "Handwritten Signature Retrieval and Identification," Pattern Recognition, vol. 17, pp. 83-90, 1996.

4] M. Hanmandlu, M.H.M. Yusof, and V.K. Madasu, "Off-line Signature Verification using Fuzzy Modeling," Pattern Recognition, vol. 38, pp. 341-356, 2005.

[5] K. Huang, and H. Yan, "Off-Line Signature Verification Based on Geometric Feature Extraction and Neural Network Classification," Pattern Recognition, vol. 30, no. 1, pp. 9-17, 1997.

[6] E.J.R. Justino, F. Bortolozzi, and R. Sabourin. "A comparison of SVM and HMM classifiers in the off-line signature verification," Pattern Recognition Letters, vol. 26, pp. 1377-1385, 2005.

[7] M.K. Kalera, S. Srihari, and A. Xu, "Off-line signature verification and identification using distance statistics," International Journal of Pattern Recognition and Artificial Intelligence, vol. 18, no. 7, pp. 1339-1360, 2004

[8] A. Kholmatov, and B. Yanikoglu, "Identity Authentication using improved online Signature Verification method," Pattern Recognition Letters, in press, 2005.

[9] X.Y. Liu and M. Blumenstein, "Experimental Analysis of the Modified Direction Feature for Cursive Character Recognition," International Workshop on the Frontiers of Handwriting Recognition (IWFHR-9), Japan, pp. 353-358, 2004.

[10] H. Lv, W. Wang, C. Wang and Q. Zhuo, "Off-line Chinese Signature Verification based on Support Vector Machines," Pattern Recognition Letters, vol. 26, pp. 2390-2399, 2005

[11] L.E. Martinez, C.M. Travieso, J.B. Alonso, and M. Ferrer, "Parametrization of a forgery Handwritten Signature Verification using SVM," IEEE 38th Annual 2004 International Carnahan Conference on Security Technology, pp. 193-196, 2004.

[12] H. Srinisvasan, M.J. Beal and S.N. Srihari, "Machine Learning approaches for Person Identification and Verification," SPIE Conference on Homeland Security, pp. 574-586, 2005

\section{Technology Review (continued from page 9)}

\section{References}

[1] T. Morgan "The DoD automated biometric identification system (ABIS)", Biometrics Task Force, [Online]. http://www.biometrics.dod.mil/documents/BRIEFS/Do D\%20ABIS_Morgan_final.pdf\#search=\%22abis\%20voice $\%$ $20 \mathrm{dna} \% 22$.

[2] J. Woodward, Jr., "Using biometrics to achieve identity dominance in the global war on terrorism", in Military Review, pp. 30-34, Sep.-Oct. 2005.

[3] "Biometrics deployment of machine readable travel documents," ICAO TAG MRTD/NTWG, Technical Report, Version 2.0, May 2004

[4] J. Lettice, (2006, Aug. 4) "How to clone the copy-friendly biometric passport," The Register [Online]. http://www.theregister.co.uk/2006/08/04/cloning_epassports/.

[5] C. Handley, "Will airports look at biometrics?" $A$ Namitech Magazine, vol. 1, pp. 13-14, 2002.

[6] Report Published by Celent, (2006, March 29) "Biometric ATMs in Japan: fighting fraud with vein pattern authentication", [Online]. http://www.celent.com/PressReleases/ 20060329(2)/BiometricsJapan.htm

[7] D. Kingsley, (2002, June 20) "Fingerprint security easy to fool," News in Science [Online]. Available: http://www.abc.net.au/science/news/stories/s585792.htm.

[8] K. Delac and M. Grgic, "A survey of biometric recognition methods," Proceedings of the 46th International Symposium Electronics in Marine, ELMAR-2004, pp. 184-193, Zadar, Croatia, 16-18 June 2004

[9] Report Published by Unisys, (Apr. 26, 2006) "Consumer worldwide overwhelmingly support biometrics for identity verification, Says Unisys Study," [Online]. Available: http://www.unisys.com/about_unisys/news_a_events/ 04268651.htm

[10] Q. Xiao, "Biometric user authentication for heightened information security," The 1st International Conference on Biometric Authentication, Lecture Notes in Computer Science, LNCS3072, pp. 708-715, Springer-Verlag, July 2004. [11] J. Jang, K. Han and J. Kim, "Evolutionary algorithmbased face verification," Pattern Recognition letters, vol. 25, pp. 1857-1865, 2004

[12] J. Jang and J. Kim, "Evolutionary pruning for fast and robust face detection", in Proc. 2006 International Joint Conference on Neural Networks, pp. 4436-4442, Vancouver, 16-21 July 2006

[13] Y. Freund and R. Schapire, "A decision-theoretic generalization of on-line learning and an application to boosting." In Proc. Second European Conference on Computational Learning Theory, Springer-Verlag, pp. 23-37, Mar. 1995.

[14] M. Herman, "Ensuring the security of America's border through the use of biometric passports and other identity documents", June 22, 2005, http://hsc.house.gov/files/ TestimonyHerman.pdf\#search=\% $\% 22 \mathrm{frvt} \% 202002 \% 20$ improvement $\% 22$

[15] K. Nakamura and S. Miyamoto, "Rotation, size and shape recognition by a spreading associative neural network," IEICE Trance. on Information and Systems, vol. E-84-D, no. 8, pp. 1075-1084, 2001

[16] K. Nakamura and H. Takano, "Rotation and size independent face recognition by the spreading associative neura network", in Proc. 2006 International Joint Conference on Neural Networks, pp. 8213-8219, Vancouver, 16-21 July 2006. [17] F. Zhao, P. Raghavan, and S. Gupta, "Automatic speech recognition in mandarin for embedded platforms," in Proc. Of ICSLP'2000, vol. 2, pp. 815-818.
[18] J. Zhang, G. Li, and W. Freeman, "Application of novel chaotic neural networks to Mandarin digital speech recognition", in Proc. 2006 International Joint Conference on Neural Networks, pp. 1380-1385, Vancouver, 16-21 July 2006. [19] M. Broomhead and D. Lowe, "Multivariable functional interpolation and adaptive networks". Complex Systems, vol. 2, pp. 321-355, 1988

[20] F. Ham, R. Acharyya, and Y. Lee, "Speaker verification using 3-D ROC curves for increasing impostor rejections", in Proc. 2006 International Joint Conference on Neural Networks, pp. 4868-4872, Vancouver, 16-21 July 2006.

[21] A. Kholmatov and B. Yanikoglu, Biometric authentication using online signatures, Lecture Notes in Computer ScienceISCIS 2004, Oct. 2004, vol: 3280, pp. 373-380.

[22] M.R. Heinen and F.S. Osório, "Handwritten signature authentication using artificial neural networks", in Proc. 2006 International Joint Conference on Neural Networks, pp. 10111-10118, Vancouver, 16-21 July 2006.

[23] H. Dozono, M. Nakakuni, H. Sanada, and Y. Noguchi, "The analysis of pen inputs of handwritten symbols using self organizing maps and its application to user authentication", in Proc. 2006 International Joint Conference on Neural Networks, pp. 4884-4889, Vancouver, 16-21 July 2006.

[24] M. Blumenstein, X.Y. Liu, and B. Verma, "A modified direction feature for cursive character recognition", International Joint Conference on Neural Networks (IJCNN '04), pp. 2983-2987, 2004.

[25] S. Armand, M. Blumenstein, and V. Muthukkumarasamy, "Off-line signature verification using the enhanced modified direction feature and neural-based classification", in Proc. 2006 International Joint Conference on Neural Networks, pp. 1663-1670, Vancouver, 16-21 July 2006. 